\section{Can we improve on situational judgement tests?}

\author{
P. Affleck, ${ }^{* 1}$ M. Bowman, ${ }_{1}^{2}$ M. Wardman, ${ }_{1}^{2}$ S. Sinclair ${ }^{3}$ and R. Adams ${ }^{2}$
}
IN BRIEF
- Provides an understanding of the selection process for dental foundation training.
- Suggests there should be a greater appreciation of the ethical concerns that underpin professional attributes.

Situational judgement tests (SJTS) are multiple-choice psychological assessments that claim to measure professional attributes such as empathy, integrity, team involvement and resilience. One of their attractions is the ability to rank large numbers of candidates. Last year SJTs formed a major component (50\% of the assessment marks) of the selection process for dental foundation training (DFT). However, it is not clear what SJTs are actually assessing. There is also the concern that applicants who have developed ethical reasoning skills may be disadvantaged by such tests. The DF selection process needs to explicitly recognise the importance of ethical reasoning.

\section{INTRODUCTION}

Dental foundation training (DFT) recruitment was centralised in 2011 and is overseen by the Committee of Postgraduate Dental Deans and Directors (COPDEND). To rank applicants COPDEND currently uses assessment centres, which eschew traditional interviews and consist of two face-to-face stations (one regarding clinical communication skills and one regarding professionalism, management and leadership skills) and a situational judgement test (SJT) paper. 50\% of the overall mark is assigned to the SJT paper. SJTs were trialled in 2013, and in 2014 the candidates were given a series of 56 questions to answer in 105 minutes. These questions each describe a dental scenario with a list of possible actions. These questions come in two varieties: ranking responses and multiple-choice. In the first, the candidate ranks five actions in order of appropriateness. In the second, the candidate chooses the three most appropriate actions. ${ }^{1}$ Failure to score highly may deny a candidate their preferred location or even jeopardise their future career (such training is a requirement for becoming an NHS performer). Anecdotally, many applicants would seem to regard the SJTs as an assessment of

"University of Leeds, Cancer Research UK Leeds Centre, Cancer Genetics Building, St. James's University Hospital, Leeds, LS9 7TF; ${ }^{2}$ School of Dentistry, ${ }^{3}$ IDEA CETL, University of Leeds, Leeds, LS2 9JT *Correspondence to: Paul Affleck Email: p.a.affleck@leeds.ac.uk

\section{Refereed Paper}

Accepted 1 December 2015

DOI: 10.1038/sj.bdj.2016.17

${ }^{\circledR}$ British Dental Journal 2016; 220: 9-10 ethical judgement, but their stated purpose is to assess professional attributes.

\section{THE NEED FOR ETHICAL REASONING}

Anyone with a contract of employment has work-related duties. However, as registered healthcare professionals, greater demands are placed upon dentists. Their professional duties are embodied in the General Dental Council's Standards for the dental team. ${ }^{2}$ This professional code sets out expected standards and is accompanied by guidance that has to be interpreted. As the document states, 'You are expected to follow the guidance, to use your professional judgement, demonstrate insight at all times and be able to justify any decision that is not in line with the guidance. ${ }^{2}$ Clearly this requires dentists to develop their ethical awareness and their ability to think through ethical dilemmas; it involves more than unreflectively following a set of rules or simply emulating more experienced colleagues.

\section{WHAT PRECISELY ARE SJTS ASSESSING?}

SJTs have the word judgement in their title so presumably some cognition, some process of reasoning, is required. However, Patterson et $a .^{3}{ }^{3}$ state the SJT, 'is designed to test non-cognitive professional attributes'. Those attributes come from the dental foundation curriculum: professional integrity, teamworking, empathy and communication, and resilience and coping with pressure. Given the time restraints, SJTs probably are a good test of coping with pressure. However, whether the aforementioned attributes are actually professional ones depends on whether they are directed by the right concerns. For example, a dentist could potentially display excellent teamworking, but towards a bad end by closely collaborating with colleagues to defraud a patient. Similarly, a dentist who is being rude to most of their patients might well be criticised for a lack of integrity. However, if the dentist believed patients deserve little respect they might show integrity by treating all of their patients rudely. The core issue here is respect for patients, and having integrity would mean treating them all with respect.

At this point it is worth looking at an actual SJT. The Patterson et al. paper in this Journal ${ }^{3}$ gives an example question of a young woman requesting veneers that do not seem to be clinically indicated (Fig. 1). A similar example is also given in the 2015 National Applicant Guide. ${ }^{4}$ Unfortunately, the model answer is not provided in either

A new patient, Louise, asks you to veneer all her front teeth. She hopes to pursue a career as a model and has been advised by a friend that veneers may help her to do this. On examination, Louise's teeth are perfectly healthy with no previous restorations and just some very mild crowding. Her teeth are a vita shade $\mathrm{A} 3$.

Rank in order the following actions in response to this situation ( $1=$ most appropriate; 5 = least appropriate):

A. Respect Louise's wishes and schedule an appointment for the veneers.

B. Ensure Louise is aware of alternatives such as orthodontics and tooth whitening.

C. Suggest a course of tooth whitening and explain the legal issues involved.

D. Establish exactly what Louise does not like about her teeth.

E. Offer to refer Louise to a specialist to discuss the veneers in more detail.

Fig. 1 Example SJT 
source, but this actually seems to be an ethical dilemma dealing with the tension between patient autonomy and acting in a patient's best interests. Communication is obviously an important issue and addressing this scenario requires ethical reflection; thus to regard it simply as a test of putatively non-cognitive professional attributes is perplexing.

The sample SJT cannot be addressed without understanding the ethical drivers behind the professional attributes of integrity, teamworking, empathy and communication. For example, a candidate may feel that a specialist referral would show teamworking and that empathy means meeting her request, with actions $\mathrm{E}$ and $\mathrm{A}$ first and second. However, if acting in the patient's best interests is given primacy, the answer could well be D, B, C, E, A.

There are other concerns with this sample SJT. For instance, the question asks what actions are most appropriate, but it would really seem to be asking in what order should you undertake these actions. There is a temporal challenge; if you start by establishing what Louise does not like about her teeth, your next action will depend on what you find out.

If we want dentists to develop ethical reasoning skills then ethical reasoning should be explicitly assessed. One option is to assess a candidate's reasoning in situations such as the Louise scenario via a third face-to-face station within the DFT selection process. The examiner would have pre-prepared prompt questions to explore the tensions between patient autonomy, professional judgement and a patient's best interests. Giving the candidate the opportunity to respond in this way would be a more valid test of their ethical reasoning and whether their decisions are being directed by the right concerns.

A further question is what constitutes an expert panel for deciding on the correct action in the dental scenario above. The 2015 Applicant Guide ${ }^{4}$ states, 'After consulting clinicians and consultants in the field, the Work Psychology Group (WPG) has written the questions for the SJTs. The WPG are experts in creating selection and assessment processes'. It would be interesting to know if the clinicians and consultants concerned have, specifically, ethical expertise.

Since SJTs are seeking to assess attributes, rather than clinical knowledge, the applicant guide states there is no need to revise for them. ${ }^{4}$ In terms of possible coaching there is a paucity of evidence in the literature regarding the effectiveness of coaching for SJTs. ${ }^{5}$ However, it strikes us as plausible that SJT scores will improve with experience of this examination format, not least in terms of pacing and answering all of the questions in the allotted time. If a student has previous experience of this format, regardless of what was being assessed, they are likely, other things being equal, to do better than their peers. This introduces an element of selection bias and may well reduce the validity of the ranking system. Therefore, to eliminate this potential source of bias, it would be sensible to provide a full mock paper. It is of note that the foundation programme for doctors, which forms the link between medical school and specialist/general practice training, provides a mock SJT paper with 70 questions accompanied by their answers, and, more importantly, the rationale for those answers. ${ }^{6}$ In comparison to their medical colleagues the dental applicants have two example questions (the example already mentioned and a further example in a presentation available on the COPDEND website $^{1}$ ) with no answers or rationale.

The SJT paper poses a number of concerns from an ethical perspective. As acknowledged by Patterson et al., ${ }^{5}$ 'It is important to note that SJTs are not measures of ethical values per se, but, rather, measures of trainees' awareness about what is effective behaviour in work-relevant contexts in important interpersonal domains'. It could be argued that SJTs reward candidates that reflexively agree with the reigning consensus. This may seem acceptable, but that consensus is likely to change over time and do we want to encourage such a passive approach from the dentists of the future? We would argue that ethical reasoning with respect to the dilemmas presented in the SJT should be encouraged.

A further concern is that having developed ethical reasoning skills, candidates may actually be at a disadvantage when taking SJTs. Students from Leeds will have been taught in seminars in which they may spend twenty or thirty minutes examining and debating a single scenario. When presented with an SJT, these candidates are likely to carefully examine each option and consider what arguments can be presented for it, however superficially implausible they may be. This would be a distinct disadvantage in a test that allows less than two minutes per question.

\section{CONCLUSION}

We argue that there is an over reliance on SJTs in the DFT selection process. It is clear that while SJTs might reflect particular ethical values they are not a valid test of ethical reasoning. Furthermore, it is not clear what SJTs are designed to test; possibly an awareness of the preferred kinds of work behaviours as determined by a panel of clinical experts. In this context, SJTs may well have a role in selecting candidates for DFT. It is plausible that if a candidate gives answers that are consistently at variance with the consensus of the expert panel, then they do not appreciate what behaviour is expected of a dental professional. However, without a mock paper with reasoned answers it is not possible to be sure SJTs are reflecting what is expected of a dental professional. We would say there is an urgent need for such a mock paper akin to the one provided to medical colleagues. In addition, we argue that the current over-reliance on SJTs may give applicants the impression that they simply require a prescribed set of professional attributes and do not need to engage in ethical reflection once they leave their dental schools.

Acknowledgements

An early version of this opinion piece was presented at an Inter-Disciplinary Ethics Applied Centre for

Excellence in Teaching and Learning seminar on the 13 May 2015. We are grateful to Dr Rob Lawlor and the other participants for their suggestions and criticisms.

1. COPDEND UK Committee of Postgraduate Dental Deans and Directors. National Recruitment to Dental Foundation Training. Available online at: http://www.copdend.org/data/files/Foundation/ Central\%20Recruitment\%20Presentation $\% 20$ for $\% 20$ August $\% 202015 \% 20-\% 2030-07-2014 \% 20$ COPDEND\%20version2.pdf (accessed November 2015).

2. GDC. Standards for the Dental Team. General Dental Council, 2013. Available online at www.gdc-uk.org/ dentalprofessionals/standards/Pages/home.aspx (accessed November 2015).

3. Patterson F, Ashworth V, Mehra S, Falcon H. Could situational judgement tests be used for selection into dental foundation training? Br Dent J 2012; 213: 23-26.

4. HESL. DFT National Applicant Guide - 2015. Available online at https://www.oriel.nhs.uk/Web/ ResourceBank/Download?file=bf3ac907-1ecc47d5-b0de-43487d5056d4.pdf\&tname=Dental $\% 20$ Foundation\%20Training\%20-\%20National\%20 Applicant\%20Guide\%202015.pdf (accessed November 2015).

5. Patterson $F$, Ashworth V, Zibarras L, Coan P, Kerrin $M, O^{\prime}$ Neill P. Evaluations of situational judgement tests to assess non-academic attributes in selection. Med Educ 2012; 46: 850-868.

6. The Foundation Programme. Situational judgement test - practice paper. Available online at www. foundationprogramme.nhs.uk/pages/home/how-toapply/SJT/EPM (accessed November 2015). 